\section{Genomic affinity in hybrids of B-genome Arachis species: new genetic resources toward peanut improvement}

\author{
Alejandra Vanina García ${ }^{1 *}$, María Celeste Silvestri ${ }^{1,4}$, Melina \\ A. Vandecaveye ${ }^{2}$, Adriana R. Custodio ${ }^{3}$, Márcio de Carvalho \\ Moretzsohn ${ }^{3}$ and Graciela I. Lavia ${ }^{1,4}$
}

\begin{abstract}
Peanut (Arachis hypogaea L.) is an important oilseed crop and food legume that is highly susceptible to biotic and abiotic factors. Wild Arachis species represent useful genetic resources because of their resistance to pests and diseases. In particular, understanding the affinity between B-genome species is important for broadening the genetic base of $A$. hypogaea (AABB genome), since only one population of $A$. ipaënsis is known, and relatively few other species have been described. Therefore, we aimed to determine the genomic affinity between A. ipaënsis and A. williamsii by developing hybrids. Microsatellite markers verified the hybrid status, and chromosome pairing and pollen viability results revealed that the species share high genomic affinity. Additionally, the presence of dyads detected in sporad and pollen analyses indicated the production of $2 n$ gametes. These results will be useful for performing evolutionary studies and developing strategies for introgression programs to improve the $B$ genome of $A$. hypogaea.
\end{abstract}

Keywords: Peanut, wild relatives, meiotic analysis, pollen viability

\section{INTRODUCTION}

Peanut (Arachis hypogaea L., Fabaceae) is one of the most cultivated legumes in the world, as it is a valuable source of oil and protein (Kamdar et al. 2020). It is an allotetraploid $(2 n=4 x=40 ; A A B B)$ originated by hybridization between $A$. duranensis Krapov. \& W.C. Greg. $(2 n=2 x=20 ; A A)$ and A. ipaënsis Krapov. \& W.C. Greg. $(2 n=2 x=20 ; B B)$, and subsequent chromosome duplication (Seijo et al. 2007, Bertioli et al. 2016), likely via sexual polyploidization (García et al. 2020).

Crop yield can be negatively affected by diseases and pests. However, wild relatives can be exploited to enhance peanut performance because they constitute natural sources of resistance (Stalker 2017). Currently, the only commercially acceptable method for improving cultivated peanut is traditional gene introgression (Cason et al. 2019), which is restricted to species of the section Arachis, as $A$. hypogaea belongs to this group. The introgression of resistance genes from wild species into $A$. hypogaea can be performed by obtaining induced hybrids from crosses between wild diploid species, duplicating chromosomes of the hybrids using colchicine, and crossing with the peanut (Simpson 1991). Since closely related species are more likely to produce fertile progenies, selecting
Crop Breeding and Applied Biotechnology 21(3): e38292139, 2021 Brazilian Society of Plant Breeding. Printed in Brazil http://dx.doi.org/10.1590/198470332021v21n3a48

\footnotetext{
*Corresponding author: E-mail: alevanina.g23@gmail.com (D) ORCID: 0000-0002-9441-0478
}

Received: 04 June 2021 Accepted: 11 August 2021 Published: 30 September 2021

${ }^{1}$ Instituto de Botánica del Nordeste (IBONE, UNNE-CONICET), W3400 Corrientes, Argentina

${ }^{2}$ Instituto Nacional de Tecnología Agropecuaria (INTA) - Estación Experimental Agropecuaria Bella Vista - Centro Regional Corrientes, W3432 Bella Vista, Corrientes, Argentina

${ }^{3}$ Embrapa Recursos Genéticos e Biotecnologia, Parque Estação Biológica, 70.770-901, Brasília, DF, Brazil

${ }^{4}$ Facultad de Ciencias Exactas, Naturales y Agrimensura (FACENA, UNNE), W3400 Corrientes, Argentina 


\section{AV García et al.}

parents is a main factor in the success of crossings. Therefore, understanding the relationships between species is crucial for the introgression and pyramiding of resistance genes in A. hypogaea and justifies more intensive studies.

Arachis ipaënsis and A. williamsii Krapov. \& W.C. Greg. ( $2 n=2 x=20 ; B B)$ are resistant to several pests, and recently conducted evaluations have shown that neither species shows symptoms of smut infection (Blas et al. 2019). Peanut smut is a soil-borne disease caused by the fungus Thecaphora frezii Carranza \& Lindquist and is currently the main economically important peanut disease in Argentina (Rago et al. 2017). Thus, the possibility of transferring some of the resistance genes from both species into the $B$ genome of peanut is of great interest. Although several crosses between $B$ genome species have been conducted, there is only one report of $A$. ipaënsis $\times A$. williamsii (Simpson and Faries 2001), and no meiotic studies have been performed to analyze their genomic homology. Meiotic studies provide relevant information on the fertility of hybrids, and therefore can accelerate the success of breeding programs (Krycki et al. 2020). Furthermore, the ability of hybrids to produce viable unreduced gametes, such as those reported in [A. duranensis $\times$ A. ipaënsis] $]^{2 \times}$ hybrids (García et al. 2020), may increase with interspecific crosses and environmental factors (Kreiner et al. 2017). Understanding this ability of Arachis hybrids is useful for inducing polyploidy in breeding programs.

Thus, we proposed the development of hybrid materials by performing reciprocal crosses between $A$. ipaënsis and $A$. williamsii, to certify hybridization using microsatellite markers, and analyze the meiotic behavior and determine the production of unreduced gametes in the $F_{1}$ hybrid progeny. We aimed to establish the relationships between these two B-genome Arachis species, considered of interest for introgression programs of resistance to key diseases in elite peanut genotypes.

\section{MATERIAL AND METHODS}

\section{Plant material}

Arachis ipaënsis, from Krapovickas et al. 30076 collection, and A. williamsii from Williams and Claire 1118 collection of the Instituto de Botánica del Nordeste (IBONE), Corrientes, Argentina, were used. The species were collected in the Tarija and Beni Departments of Bolivia, respectively, and are $2 n=2 x=20$ with B genome. The hybrids obtained [A. ipaënsis $\times A$. williamsii $]^{2 \mathrm{x}}$ and $[A \text {. williamsii } \times A \text {. ipaënsis }]^{2 \mathrm{x}}$ are referred to here as IpaWil and Willpa, respectively.

\section{Crosses and hybrid identification}

Reciprocal crosses were performed between October 2015 and April 2016 in a greenhouse, according to the methods of Favero et al. (2006). During June and July 2016, the fruits were harvested and stored in the Germplasm Bank of the IBONE, and in September, the seeds were placed in individual germinators.

To identify true hybrid individuals, the seedlings were analyzed using microsatellite markers. The markers were developed by different authors, but the primer sequences, references, and other information were compiled on PeanutBase (https://peanutbase.org/search/marker). Total DNA was extracted according to the method described by Inglis et al. (2018). DNA amplification reactions were performed by multiplex PCR using until three fluorescences (6FAM, HEX, and NED) by reaction and similar annealing temperatures. The PCR of microsatellite loci was performed as described by Moretzsohn et al. (2013). For allele sizing, the electropherograms were analyzed using the GeneMapper ${ }^{\circledR}$ software (Applied Biosystems).

\section{Meiotic and pollen analysis}

Pollen mother cells (PMCs) from young flower buds were stained in $2 \%$ lactopropionic orcein, and the frequency of all PMCs with normal and abnormal behavior was recorded from prophase I to telophase II. In addition, sporads colored with carmine:glycerin (1:1) were analyzed. A minimum of 300 sporads per flower bud was counted for each hybrid combination. The meiotic index was calculated as follows:

$\% M I=[($ number of normal tetrads $\times 100) /$ total of post meiotic products counted] (Love 1951). Sporads with four microspores of the same size were considered normal tetrads, and any deviation was considered abnormal. The presence of dyads, triads, and monads was recorded as evidence of the production of $2 n$ and $4 n$ gametes. 
Pollen viability was estimated in three flowers from each individual hybrid using the same technique as described for sporads. Regularly shaped and darkly stained pollen grains were considered viable and counted using ImageJ software (Rasband 1997). Pollen viability $=$ (stained pollen grains $\div$ total pollen grains $) \times 100$. Viable pollen grains from progenitors were measured with ImageJ software, using the Em index (Van Campo 1957). The production of unreduced pollen in the hybrids was detected following the Darlington (1937) criteria: pollen grains 1.25 times larger than the average size of each individual hybrid were considered as large $2 n$, whereas pollen grains 0.25 times smaller than the average size were described as small (García et al. 2020). Accordingly, viable pollen grains were classified as normal, small, or large $2 \mathrm{n}$. Pollen size data were not normally distributed; therefore, we ran a one-way non-parametric ANOVA (Kruskal-Wallis test) ( $\alpha$ level $=0.05$ ) to evaluate the existence of significant differences among pollen sizes from $A$. ipaënsis, $A$. williamsii, IpaWil, and Willpa. Multiple a posteriori comparisons were calculated between the mean ranks of each taxon using Dunn's test. Statistical analyses were performed using R software v 3.5.3 (R Core Team 2019).

\section{RESULTS AND DISCUSSION}

\section{Crosses and hybrid identification}

Only one accession of $A$. ipaënsis is known. Thus, understanding the crossability and relationships between the B-genome species is necessary to broaden the genetic base of $A$. hypogaea in breeding programs. In this study, $A$. ipaënsis was used as the female parent in 158 pollinations (44.89\%), with peg development in $48.73 \%$, whereas $A$. williamsii was the female parent in 194 crosses (55.11\%), forming pegs in $25.26 \%$ of cases. After harvest, 66 seeds were obtained from A. ipaënsis $\times A$. williamsii crosses and 44 seeds were collected from the reciprocal crosses. Although the formation of pegs and seeds does not guarantee hybrid development, values were high in both crosses, with greater returns when $A$. ipaënsis was the female parent. Custodio et al. (2021) obtained greater fruit numbers and success rates for $A$. gregoryi $\times$ A. williamsii crosses than for A. gregoryi $\times$ A. ipaensis. Similarly, Wondracek-Lüdke et al. (2015) obtained higher values of these parameters for $A$. valida $\times A$. williamsii than for $A$. valida $\times A$. ipaënsis. Considering the results of our crosses, $B$ genome species with many collected accessions, such as $A$. gregoryi, $A$. magna, and $A$. valida, could use $A$. williamsii as a bridge species with the $B$ genome of $A$. hypogaea, which has $99.96 \%$ homology with the $A$. ipaënsis genome (Bertioli et al. 2016). However, hybrids obtained in the studies mentioned above had low or null fertility, suggesting that more relationship studies between $B$ genome species are required to evaluate this possibility.

Only seedlings obtained from seed germination were used in the analyses; the remaining seeds were stored in the Arachis Germplasm Bank of IBONE. A total of 47 microsatellite markers were screened for polymorphism against both parents and 18 were informative (PM36, PM45, PM73, PM170, PM210, pPGPSeq2H11, pPGSseq14H6, pPGSseq16C6, RN8C09, RN20C10, RN23A07, AC2B05, TC3E05, TC4G02, TC7E04, IPHAM108, GM1989, and Ad_FI308518). The 18 polymorphic markers confirmed that four of the eight Willpa seedlings were true $F_{1}$ hybrids, three were self-pollinated, and one was the result of pollination by an unknown male parent. Of the four IpaWil seedlings, three were true $F_{1}$ hybrids and one was self-pollinated. Microsatellite markers were chosen because of their codominant nature, and the ability to identify the seedlings as truly hybrid, self-pollinated, or undesirably cross-pollinated. Selfed and contaminated seedlings were discarded, and seven true hybrids were analyzed.

\section{Meiotic analysis}

In Arachis, crossability studies have been useful at the genus level for grouping species into nine taxonomic sections (Krapovickas and Gregory 1994), and chromosome pairing in $F_{1}$ hybrids has been used to trace affinities and group species (Singh and Moss 1984). We analyzed chromosomal pairing in metaphase I and meiotic behavior from prophase I to telophase II.

\section{Chromosome pairing}

The analysis of 175 and 133 PMCs of IpaWil and Willpa hybrids, respectively, revealed five meiotic configurations, including univalent (I), bivalent (II), trivalent (III), and quadrivalent (IV) associations. The most frequent association was bivalent, averaging 9.63 in IpaWil and 9.44 in Willpa, while univalents ranged from zero to four per PMC, with means of 0.32 and 0.54 , respectively. Multivalents were observed at very low frequencies in the two hybrid combinations. The 
meiotic configuration 10II (Figure 1A) was the most common, with values of $83.43 \%$ in IpaWil and $76.69 \%$ in Willpa, while 9 II+2I (Figure 1B) represented $9.14 \%$ and $15.04 \%$ of the PMC analyzed in the respective hybrids, and the remaining three configurations $(8 I I+4 I, 8 I I+1 I I+1 I$, and $8 I I+1 I V$; Figures $1 C$ and $D)$ accounted for less than $8 \%$ of the cells.

In cytological studies performed on Arachis intragenomic hybrids between A-genome species, the mean bivalent association ranged from 9.1 to 9.9, while the number of univalents ranged from zero to four per PMC with means from 0.05 to 1.35 (Singh and Moss 1984, Stalker et al. 1991). Tallury et al. (2005) reported mean values of 0.3 univalents and 9.9 bivalents in hybrids between $\mathrm{K}$ genome species. Although there are no reports of meiotic behavior in hybrids between $B$ genome species, the hybrids analyzed in this study presented high frequencies of bivalents, with low or null presence of univalents. Closely related species are expected to have a high number of bivalents (Stalker et al. 1991),

Table 1. Meiotic abnormalities and sporads in hybrids between A. ipaënsis and A. williamsii

\begin{tabular}{|c|c|c|c|c|c|c|c|}
\hline Phases & Meiotic abnormalities & \multicolumn{3}{|c|}{ IpaWil } & \multicolumn{3}{|c|}{ Willpa } \\
\hline $\mathrm{PI}$ & Cytomixis & 141 & 5 & 3.55 & 583 & 227 & 47.51 \\
\hline \multirow[t]{2}{*}{ MI } & Out of plate chromosomes & 749 & 188 & 25.10 & \multirow[t]{2}{*}{395} & 103 & 26.08 \\
\hline & Cytomixis & & 15 & 2.00 & & 33 & 8.35 \\
\hline \multirow[t]{2}{*}{$\mathrm{TI}$} & Cytomixis & 279 & 52 & 18.64 & \multirow[t]{2}{*}{120} & - & - \\
\hline & Out of nucleus chromosomes & & 28 & 10.03 & & - & - \\
\hline \multirow[b]{3}{*}{ PII } & Normal & & 136 & 62.10 & \multirow[b]{3}{*}{114} & 47 & 41.23 \\
\hline & Out of nucleus chromosomes & & 7 & 3.20 & & - & - \\
\hline & Unbalanced nuclei & 219 & 62 & 28.31 & & 63 & 55.26 \\
\hline \multirow{4}{*}{ MII } & Out of plate chromosomes & & 13 & 21.31 & \multirow{4}{*}{29} & 5 & 17.24 \\
\hline & Bridges & 61 & 1 & 1.64 & & 1 & 3.45 \\
\hline & Nucleus of restitution & & 15 & 24.59 & & - & - \\
\hline & Cytomixis & & - & - & & 1 & 3.45 \\
\hline \multirow{6}{*}{ TII } & Normal & \multirow{6}{*}{1137} & 783 & 68.87 & \multirow{6}{*}{166} & 87 & 52.41 \\
\hline & Unbalanced nuclei & & 24 & 2.11 & & 42 & 28 \\
\hline & Multiple nuclei & & - & - & & 3 & 1.81 \\
\hline & Micronuclei & & 7 & 0.62 & & - & - \\
\hline & 1 nucleus of restitution $2 n$ & & 61 & 5.36 & & 18 & 10.84 \\
\hline & Cytomixis & & 262 & 23.04 & & - & - \\
\hline Total & & 2623 & & & \multicolumn{3}{|l|}{1407} \\
\hline Abnormal Triads & & & 11 & 0.40 & & 9 & 0.32 \\
\hline Dyads & & & 75 & 2.73 & & 195 & 7.12 \\
\hline Abnormal Dyads & & & 30 & 1.09 & & 445 & 16.24 \\
\hline Monads & & & - & - & & 1 & 0.05 \\
\hline Abnormal Monads & & & 5 & 0.18 & & - & - \\
\hline \multirow[t]{2}{*}{ Total } & & \multicolumn{3}{|c|}{2745} & \multicolumn{3}{|c|}{2740} \\
\hline & $\% \mathrm{MI}$ & \multicolumn{3}{|c|}{80.22} & & 64.38 & \\
\hline
\end{tabular}

Phases: P, prophase; M, metaphase; A, anaphase; T, telophase; I, first meiotic division; II, second meiotic division. MI, meiotic index. 
and a high frequency of bivalents in intragenomic hybrids suggests strong genomic similarities between species (Singh and Moss 1984).

Multivalents have only been reported in a few Arachis hybrids and at low frequencies (Singh and Moss 1984, Stalker et al. 1991). Quadrivalents could occur due to the presence of reciprocal translocation heterozygotes, by which cytological differentiation and subsequent speciation develop among Arachis taxa (Stalker et al. 1991). According to Singh and Moss (1984), a reduction in bivalent and an increase in univalent frequency in hybrids indicate reduced homology between parental chromosomes, whereas a decrease in bivalents and the formation of multivalents indicate a repatterning of genetic material and not a reduction in homology. Therefore, both the low degree of multivalent association and the high level of bivalent association observed in this study are not conclusive to generalize chromosome structural differentiations and clearly show a high degree of homology between the B genomes of $A$. ipaënsis and $A$. williamsii.
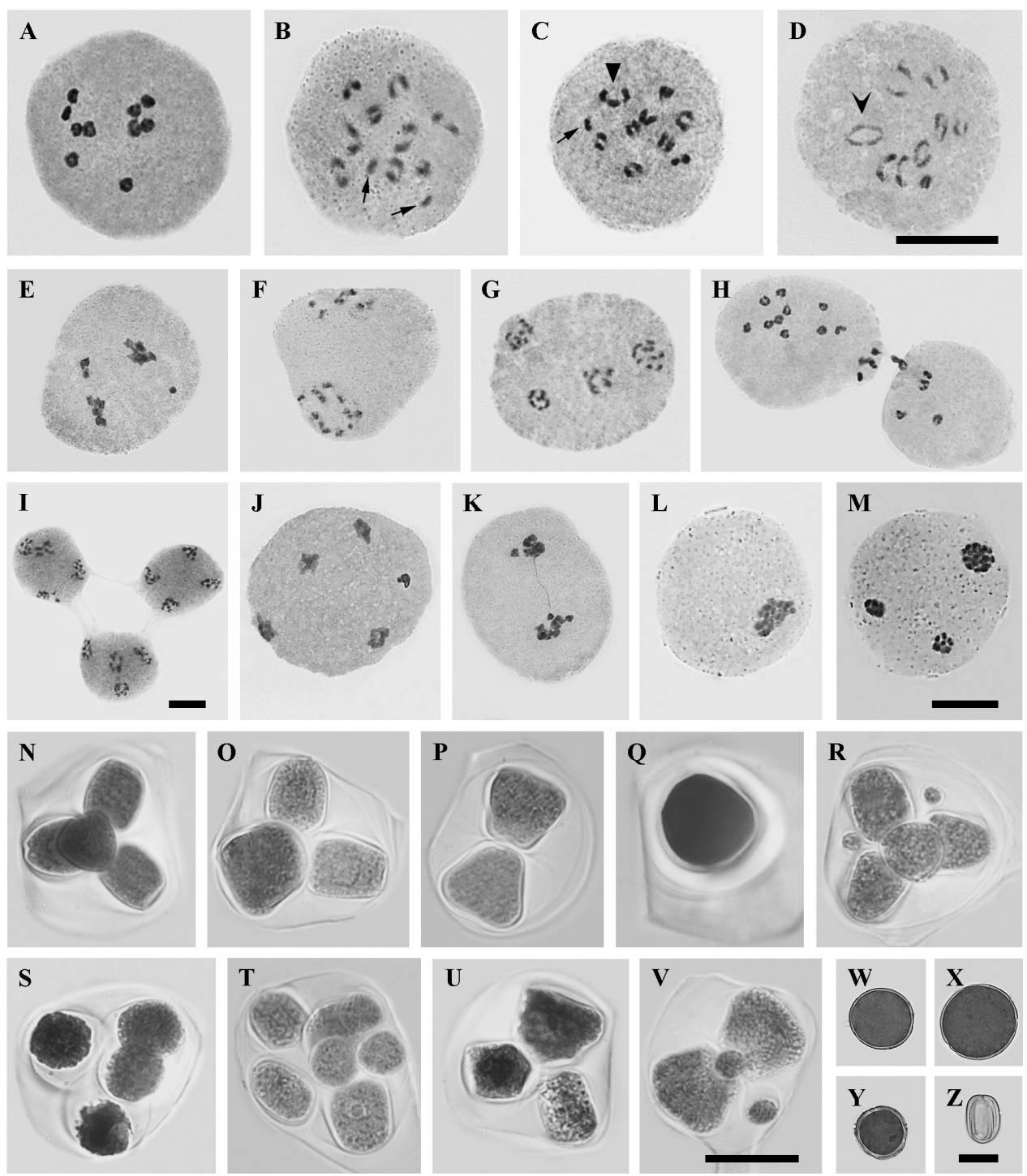

Figure 1. Meiotic analysis in hybrid individuals. Meiotic configurations: $10 I I(A) ; 9 I I+2 I$ (B); $8 I I+1 I I I+1 I$ (C); $8 I I+1 I V$ (D). Meiotic irregularities: MII with out-of-plate chromosomes (E); PII and TII with unbalanced nuclei (F-G); Di with cytomixis (H); TII with cytomictic channels (I); TII with multiple nuclei (J); MII with bridge (K); MII and TII with restitution nucleus (L-M). Sporads: tetrad (N); triad (O); dyad (P); monad (Q); abnormal tetrads (R-S); polyad (T); abnormal triad (U) and abnormal dyad (V). Pollen: normal (W); $2 \mathrm{n}(\mathrm{X})$; small (Y); unviable (Z). P, prophase; Di, diakinesis; M, metaphase; A, anaphase; T, telophase; I, first meiotic division; II, second meiotic division. Arrow $=$ univalent. Triangle $=$ trivalent. Arrowhead $=$ tetravalent. Scale bar $=20 \mu \mathrm{m}$. 


\section{Chromosome behavior}

The analysis of IpaWil and Willpa hybrids revealed a rate of regularity of meiotic division in $71.26 \%$ and $59.77 \%$ of the PMCs, respectively. The irregularities detected included out-of-plate and out-of-nucleus chromosomes that were found at high frequencies in metaphases I and II, telophase I, and prophase II (Table 1, Figure 1E). These irregularities could explain the cells with micronuclei in telophase II or those with unbalanced nuclei in prophase II and telophase II (Figure $1 \mathrm{~F}-\mathrm{G}$ ), and may also be related to the presence of cytomixis, as this phenomenon was detected in the majority of phases of meiotic division (Figure $1 \mathrm{H}-\mathrm{I}$ ). We observed empty cells, suggesting that they had undergone cytomixis and transferred all of the genetic material (unrecorded data). This could also explain cells with a larger number of nuclei than normally expected in telophase II (Figure 1J). Bridges were the irregularities less often found in metaphase II (Figure $1 \mathrm{~K})$. Finally, restitution nuclei occurred in metaphase II (Figure $1 \mathrm{~L}$ ), and cells with two haploid nuclei and a $2 \mathrm{n}$ restitution nucleus were found in telophase II (Figure 1M).

Meiotic irregularities have been related to the origin of aneuploid gametes (Mursalimov and Deineko 2018), which impose detrimental effects on pollen development (Shin et al. 2021). Out-of-plate and out-of-nucleus chromosomes can be caused by unequal tension from the poles (Lampson and Grishchuk 2017). Meanwhile, cytomixis is considered an additional mechanism for the origin of aneuploidy and polyploidy, since, when only a part of the genome is transferred, unbalanced and sterile gametes are formed (García et al. 2020). Similarly, unequal separation of chromosomes to the poles results in the formation of micronuclei (Zhang et al. 2018). In our analysis, micronuclei were observed at a low frequency compared to the percentage of PMCs with abnormalities; therefore, most of the irregularly segregated chromosomes were likely included in at least one of the main nuclei. Nevertheless, such inclusions do not assure the restitution of the euploid chromosome number of the main nucleus (García et al. 2020), which could explain the unbalanced nuclei observed in telophase II. The occurrence of multiple nuclei in telophase II may relate to the presence of multipolar spindles, as has been observed in other genera (Reis et al. 2016).

Restitution nuclei in PMCs in metaphase II of IpaWil hybrids could have arisen due to processes of nuclear restitution that began in the first meiotic division. During anaphase II and telophase II, these nuclei could continue the meiotic division resulting in two $2 \mathrm{n}$ nuclei, or undergo a second nuclear restitution, to produce $4 \mathrm{n}$ nuclei (not shown). In addition, processes of nuclear restitution can originate during the second meiotic division and produce a restitution nucleus and two haploid nuclei, similar to those observed in telophase II in both hybrids.

\section{Sporad analysis}

The analysis showed that IpaWil hybrids produced a high number of tetrads (Figure $1 \mathrm{~N}$ ) and consequently a high meiotic index (MI) (80.22\%) with low percentages of abnormal spores, including sporads associated with the formation of unreduced gametes such as triads and dyads (Table 1). In Willpa hybrids, the analysis revealed an intermediate MI (64.38\%), likely due to a higher proportion of abnormal sporads, including triads, dyads, and monads (Table 1, Figure 10-Q). The meiotic index (MI) allows for the assessment of the regularity of the meiotic process, since a higher MI indicates a more normal meiotic chromosome behavior (Love 1951). In our results, the Mls of hybrids were higher than the meiosis regularity values, which indicate that irregularities do not generally interfere with the normal process of pollen grain formation. In addition, the abnormalities observed in meiosis were consistent with the abnormal sporads found. For instance, the presence of irregularities in telophase II would lead to the formation of abnormal tetrads or polyads (Figure $1 \mathrm{R}-\mathrm{T}$ ), and thus to the formation of abnormal gametes and the reduction of viability. The abnormalities detected in IpaWil hybrid sporads would affect not only the proportion of normal n-pollen grains, but also the production of unreduced gametes, as abnormal triads, dyads, and monads were present (Figure $1 \mathrm{U}-\mathrm{V}$ ).

Regarding sporads related to the formation of unreduced gametes, the presence of cells with $2 \mathrm{n}$ restitution nuclei in telophase II explains the occurrence of triads. Although the meiotic mechanisms involved were not determined, the detection of dyads and monads indicated the occurrence of unreduced gametes. Hybrids between $A$. duranensis and $A$. ipaënsis have recently been shown to present tripolar spindles and cytomixis, which lead to the formation of restitution nuclei. Consequently, these failures contribute to the production of triads and dyads and ultimately $2 \mathrm{n}$ pollen grains (García et al. 2020). 


\section{Pollen analysis}

The pollen grains of $A$. ipaënsis, $A$. williamsii, and the seven hybrids obtained were analyzed to estimate pollen viability (PV) and determine the presence of unreduced gametes based on the size and morphology of pollen grains. Both parental species had high PV (A. ipaënsis $98.75 \%$ and $A$. williamsii, $96.20 \%)$. Pollen viability of hybrids is a traditional tool used in the genus Arachis to infer genomic relationships between parental species. It is accepted that intraspecific, intragenomic, and intergenomic hybrids show pollen viability values greater than $70 \%$, between $25 \%$ and $70 \%$, and below 10\%, respectively (Stalker et al. 1991, Krapovickas and Gregory 1994). The average PV of IpaWil was $70.19 \%$, whereas that of Willpa was $64.99 \%$ (Table 2). These values are in agreement with the number of normal tetrads found, as reported in hybrids of other genera (Fernandes Moreira et al. 2017). Simpson and Faries (2001) reported that hybrids from crosses between $A$. williamsii and $A$. ipaënsis had a pollen stainability of $62.9 \%$. These data support that $A$. ipaënsis and $A$. williamsii have similar genomes, which was confirmed by our analysis of bivalent formation. Simpson and Faries (2001) also reported that hybrids between A. magna (B genome) and A. ipaënsis had a $90.6 \%$ PV. In contrast, hybrids between $A$. valida (B genome) and the other B genome species showed PV varying from $10.5 \%$ with $A$. gregoryi to $39.4 \%$ with $A$. magna (Wondracek-Lüdke et al. 2015). Hybrids between $A$. gregoryi and the other B genome species showed lower values, from $2.6 \%$ with $A$. williamsii to $10.45 \%$ with A. magna (Simpson and Faries 2001, Custodio et al. 2021). These results suggest that the $B$ genome group has a subgroup of further related species composed of $A$. ipaënsis, $A$. magna, and $A$. williamsii, whose hybrids show pollen viability values above $62 \%$. Thus, although $B$ genome species form a uniform group (Bechara et al. 2010, Robledo and Seijo 2010, Moretzsohn et al. 2013), differences between their karyotypes could influence the fertility of their hybrids. The three species mentioned share the same karyotype formula, and two ribosomal loci on pair 3 are present only in A. magna and A. ipaënsis; in contrast, a distal DAPI band on pair 3 was observed in A. gregoryi, A. valida, and A. williamsii, which could reflect a closer relationship among these taxa (Robledo and Seijo 2010). Therefore, new crosses among all B genome species, including meiotic analysis, could better define their homology, as crossings between $A$. magna and A. williamsii are still lacking.

Regarding the size of viable pollen grains, a statistically significant difference was detected among all genotypes: $A$. ipaënsis, A. williamsii, IpaWil, and Willpa (Kruskal-Wallis test, Dunn test, $\mathrm{p}$ values $<0.001$ ). The average pollen size in A. williamsii was $31.48 \pm 1.59 \mu \mathrm{m}$, varying from 26.73 to $36.13 \mu \mathrm{m}$, whereas that in A. ipaënsis was $32.19 \pm 1.79 \mu \mathrm{m}$, varying from 27.28 to 35.80 . The hybrids, particularly Willpa, showed wider size ranges and larger pollen sizes than the parental species. The average pollen size in IpaWil was $33.02 \pm 4.36 \mu \mathrm{m}$, varying from 20.83 to $46.11 \mu \mathrm{m}$, whereas that in Willpa and $35.04 \pm 6.50 \mu \mathrm{m}$, varying from 20.02 to $49.80 \mu \mathrm{m}$ (Figure 2).

Pollen viability values are consistent with the high production of viable grains of different sizes, since in addition to normal pollen grains, we observed unreduced pollen grains $(2 n)$ and small size grains (Figure $1 W-Y$ viable; $Z$ unviable). Unreduced pollen grains were considered with a size equal to or larger than 41.27 and $43.80 \mu \mathrm{m}$ in IpaWil and Willpa

Table 2. Types of pollen grains found and pollen viability evaluated in hybrids

\begin{tabular}{|c|c|c|c|c|c|c|}
\hline \multirow{2}{*}{ Individual } & \multirow{2}{*}{$n$} & \multicolumn{3}{|c|}{ Viable (\%) } & \multirow[t]{2}{*}{ Non-viable (\%) } & \multirow[t]{2}{*}{ Viability (\%) } \\
\hline & & No & $2 n$ & Sm & & \\
\hline A. ipaënsis & 1500 & - & - & - & 1.25 & 98.75 \\
\hline A. williamsii & 1500 & - & - & - & 3.80 & 96.20 \\
\hline \multicolumn{7}{|l|}{ IpaWil } \\
\hline 1 & 1500 & 56.13 & 0.67 & 13.40 & 29.80 & 70.20 \\
\hline 2 & 1500 & 56.86 & 0.40 & 11.13 & 31.61 & 68.39 \\
\hline 6 & 2540 & 64.96 & 0.98 & 6.06 & 28.00 & 72.00 \\
\hline mean & & 59.31 & 0.68 & 10.19 & 29.80 & 70.19 \\
\hline \multicolumn{7}{|l|}{ Willpa } \\
\hline 4 & 2665 & 57.15 & 1.80 & 5.82 & 35.23 & 64.77 \\
\hline 5 & 2723 & 59.09 & 0.26 & 7.27 & 33.38 & 66.62 \\
\hline 7 & 2847 & 58.90 & 1.23 & 4.18 & 35.69 & 64.31 \\
\hline 8 & 2159 & 63.55 & 0.70 & 7.78 & 27.97 & 64.25 \\
\hline mean & & 59.67 & 0.99 & 6.26 & 33.08 & 64.99 \\
\hline
\end{tabular}

$n$, number of pollen grains analyzed; No, reduced normal pollen grain; $2 n$, giant pollen grain; Sm, small size pollen. 


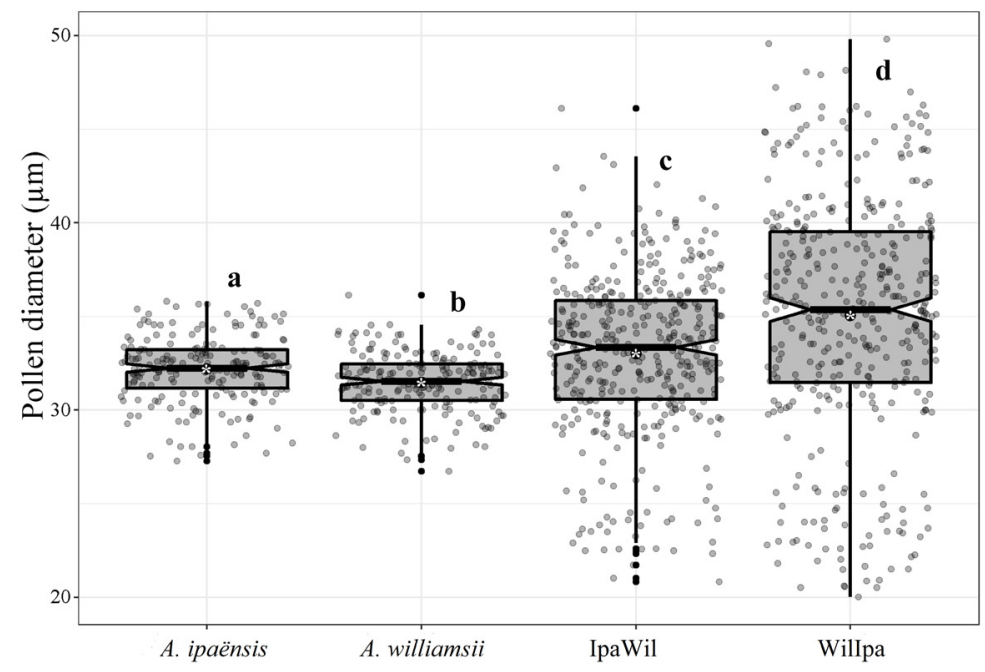

Figure 2. Comparison of pollen size data among $A$. ipaënsis, $A$. williamsii, Willpa and IpaWil. In each boxplot, the box represents the middle $50 \%$ of scores (Q1-Q3), the horizontal line represents the median, the notches represent the $95 \%$ confidence interval for the median, the white asterisk represents the mean, and the gray dots represent the values observed. The lower whisker extends to the lowest sample item not more than 1.5xIQR (Q3 - Q1) below Q1, whereas the upper whisker extends to the largest sample item not more than 1.5xIQR above Q3. The individual black dots outside the whiskers are the outliers. Different letters indicate a statistically significant difference (Kruskal-Wallis, Dunn tests, $p<0.001$ ). Additionally, the notches from different boxes that do not overlap show that there are significant differences between the pollen sizes compared. It is noticeable that the values of $A$. ipaënsis are higher than those of $A$. williamsii, and that the hybrids have a wider size range than parental species.

hybrids, respectively. The production of unreduced pollen grains was lower in the former but varied among individuals in both crosses (Table 2). Previous data have shown that the frequency of $2 n$ gamete production could vary among species, cultivars, and even among flowers of an individual (Kreiner et al. 2017). Our results showed production between $0.40 \%$ and $0.98 \%$ of unreduced gametes in IpaWil, and $0.26 \%$ to $1.80 \%$ in Willpa (Table 2 ). These gametes were probably the product of the restitution nuclei formed because of the non-disjunction of chromosomes in anaphase I and anaphase II. The presence of unreduced pollen grains together with dyads and triads evidenced that these hybrids can form unreduced gametes and that they could naturally generate polyploid individuals. Our data, together with a recent report by García et al. (2020), suggest that the production of $2 n$ gametes by interspecific $F_{1}$ hybrids of Arachis is not an isolated event in this genus. The production of unreduced gametes is useful for improving crop species by enabling crosses between plants of different ploidy levels (Brownfield and Köhler 2011). Therefore, the analysis of the production and frequency of unreduced gametes in Arachis hybrids provides useful information that could be used for breeding programs. This is important considering that the method to detect unreduced gametes is inexpensive and rapid, and provides information on the reproductive biology of Arachis species hybrids. For example, the IpaWil and Willpa hybrids studied here are sufficiently fertile to produce seeds. In addition, as they produce unreduced gametes, they would likely generate natural polyploids in subsequent generations.

In summary, we developed hybrid materials between $A$. ipaënsis and A. williamsii, analyzed the meiotic behavior, and confirmed the production of $2 n$ gametes in the $F_{1}$ hybrid progeny. Our results on chromosome pairing and pollen viability confirm that the two species share high genomic affinity. Furthermore, the number of seeds obtained in the crosses, meiotic index, meiotic chromosome behavior, and pollen viability appear to indicate that reproductive success is higher when $A$. ipaënsis is used as the female parent. All data provided will be useful for performing evolutionary studies and for introgression programs to improve the B genome of $A$. hypogaea.

\section{ACKNOWLEDGEMENTS}

Our thanks to projects: ANPCYT (PICT-2015-2681) and SGCyT-UNNE (PI 6P003). 
Genomic affinity in hybrids of B-genome Arachis species: new genetic resources toward peanut improvement

\section{REFERENCES}

Bechara MD, Moretzsohn MC, Palmieri DA, Monteiro JP, Bacci M, Martins $J$ and Gimenes MA (2010) Phylogenetic relationships in genus Arachis based on ITS and 5.8 S rDNA sequences. BMC Plant Biology 10: 255.

Bertioli DJ, Cannon SB, Froenicke L, Huang G, Farmer AD, Cannon EKS, Liu X, Gao D, Clevenger J, Dash S, Ren L, Moretzsohn MC, Shirasawa K, Huang W, Vidigal B, Abernathy B, Chu Y, Niederhuth CE, Umale $P$, Araújo ACG, Kozik A, Do Kim K, Burow MD, Varshney RK, Wang $X$, Zhang X, Barkley N, Guimarães PM, Isobe S, Guo B, Liao B, Stalker HT, Schmitz RJ, Scheffler BE, Leal-Bertioli SCM, Xum X, Jackson SA, Michelmore $R$ and Ozias-Akins $P$ (2016) The genome sequences of Arachis duranensis and Arachis ipaënsis, the diploid ancestors of cultivated peanut. Nature Genetics 48: 438-446.

Blas FJ, Bressano M, Teich I, Balzarini MG, Arias RS, Manifesto MM and Buteler MI (2019) Identification of smut resistance in wild Arachis species and its introgression into peanut elite lines. Crop Science 59: 1657-1665.

Brownfield L and Köhler C (2011) Unreduced gamete formation in plants: mechanisms and prospects. Journal of Experimental Botany 62:1659-1668.

Cason JM, Simpson CE, Rooney WL and Brady JA (2019) First report of successful hybridization between Arachis vallsii and A. dardani. Agrosystem, Geosciences \& Environmental 2: 190013.

Custodio AR, Simpson CE and Valls JFM (2021) Crossability between Arachis gregoryi (Fabaceae) and wild Arachis species with distinct genomes. Bonplandia 30: 91-106

Darlignton CD (1937) Recent advances in cytology. Churchill, London, $671 p$.

Favero AP, Simpson CE, Valls JFM and Vello NA (2006) Study of the evolution of cultivated peanut through crossability studies among Arachis ipaënsis, A. duranensis, and A. hypogaea. Crop Science 46: 1546-1552.

Fernandes Moreira N, Santana Pereira TN and Coutinho Martins K (2017) Meiotic analysis of interspecific hybrids between Capsicum frutescens and Capsicum chinense. Crop Breeding and Applied Biotechnology 17: 159-163.

García AV, Ortiz AM, Silvestri MC, Custodio AR, Moretzsohn MC and Lavia GI (2020) Occurrence of $2 n$ microspore production in diploid interspecific hybrids between the wild parental species of peanut (Arachis hypogaea L., Leguminosae) and its relevance in the genetic origin of the cultigen. Crop Science 64: 2420-2436.

Inglis PW, Pappas MdCR, Resende LV and Grattapaglia D (2018) Fast and inexpensive protocols for consistent extraction of high quality DNA and RNA from challenging plant and fungal samples for highthroughput SNP genotyping and sequencing applications. PloS One 13: e0206085.

Kamdar JH, Jasani MD, Ajay B, Kumar S and George JJ (2020) Effect of selection response for yield related traits in early and later generations of groundnut (Arachis hypogaea L.). Crop Breeding and

\section{Applied Biotechnology 20: e317320215}

Krapovickas A and Gregory WC (1994) Taxonomía del género Arachis (Leguminosae). Bonplandia 8: 1-186.

Kreiner JM, Kron P and Husband BC (2017) Evolutionary dynamics of unreduced gametes. Trends in Genetics 33: 583-593.

Krycki KC, Tedesco M, Rodrigues V, Weiler RL, Brunes AP, Dall'Agnol M and Simioni C (2020) Reproductive analyses of intraspecific Paspalum notatum Flügge hybrids. Crop Breeding and Applied Biotechnology 20: e291620114.

Lampson MA and Grishchuk EL (2017) Mechanisms to avoid and correct erroneous kinetochore-microtubule attachments. Biology 6: 28067761

Love RM (1951) Varietal differences in meiotic chromosomes behavior of Brazilian wheats. Agronomy Journal 43: 72-76.

Moretzsohn MC, Gouvea EG, Inglis PW, Leal-Bertioli SC, Valls JF and Bertioli DJ (2013) A study of the relationships of cultivated peanut (Arachis hypogaea) and its most closely related wild species using intron sequences and microsatellite markers. Annals of Botany 111: $113-126$.

Mursalimov S and Deineko E (2018) Cytomixis in plants: facts and doubts. Protoplasma 255: 719-731.

R Core Team (2019) R: A language and environment for statistical computing. R Foundation for Statistical Computing, Vienna. Available at <https://www.R-project.org/>. Accessed on May 2, 2020.

Rago AM, Cazón LI, Paredes JA, Molina JPE, Conforto EC, Bisonard E and Oddino C (2017) Peanut smut: from an emerging disease to an actual threat to Argentine peanut production. Plant Disease 101: 400-408

Rasband WS (1997) ImageJ. Bethesda, MD: National Institutes of Health. Available at <http://imagej.nih.gov/ij>. Accessed on May 9, 2020.

Reis AC, Sousa SM and Viccini LF (2016) High frequency of cytomixis observed at zygotene in tetraploid Lippia alba. Plant Systematics and Evolution 302: 121-127.

Robledo GA and Seijo JG (2010) Species relationships among the wild B genome of Arachis species (section Arachis) based on FISH mapping of rDNA loci and heterochromatin detection: A new proposal for genome arrangement. Theoretical and Applied Genetics 121: 1033-1046.

Seijo JG, Lavia GI, Fernandez A, Krapovickas A, Ducasse DA, Bertioli DJ and Moscone EA (2007) Genomic relationships between the cultivated peanut (Arachis hypogaea, Leguminosae) and its close relatives revealed by double GISH. American Journal of Botany 94: 1963-1971.

Shin H, Park HR, Park JE, Yu SH, Yi G, Kim JH, Koh W, Kim HH, Lee S and Huh JH (2021) Reduced fertility caused by meiotic defects and micronuclei formation during microsporogenesis in xBrassicoraphanus. Genes \& Genomics 43: 251-258

Simpson CE (1991) Use of wild Arachis species/introgression of genes into A. hypogaea L. Peanut Science 28: 114-116. 


\section{AV García et al.}

Simpson CE and Faries MW (2001) Advances in the characterization of diversity in section Arachis: archeological evidence, crossing results and their relationship in the understanding the origins of Arachis hypogaea L. Simpósio de recursos genéticos para a América a Latina e Caribe. Instituto Agronómico de Paraná, Londrina, p.103-104.

Singh AK and Moss JP (1984) Utilization of wild relatives in genetic improvement of Arachis hypogaea L. Part 5. Genome analysis in section Arachis and its implications in gene transfer. Theoretical and Applied Genetics 68: 355-364.

Stalker HT (2017) Utilizing wild species for peanut improvement. Crop Science 57: 1102-1120.

Stalker HT, Dhesi JS, Parry DC and Hahn JH (1991) Cytological and interfertility relationships of Arachis section Arachis. American
Journal of Botany 78: 238-246.

Tallury S, Hilu K, Milla S, Friend S, Alsaghir M, Stalker HT and Quandt D (2005) Genomic affinities in Arachis section Arachis (Fabaceae): Molecular and cytogenetic evidence. Theoretical and Applied Genetics 111: 1229-1237.

Van Campo M (1957) Palynologie africaine I. Bulletin de l'Institut Francais d'Afrique Noire 19: 659-678.

Wondracek-Lüdke DC, Custodio AR, Simpson CE and Valls JFM (2015) Crossability of Arachis valida and B genome Arachis species. Genetic and Molecular Research 14: 17574-17586.

Zhang W, Wang C, Xue L, Zheng Y and Lei J (2018) Production of pollen less triploid lily hybrids from Lilium pumilum DC. ×'Brunello'. Euphytica 214: 171. 